\title{
Signs of Hypothetical Flora and Fauna of the Planet Venus: Returning to Archive of the Old TV-Experiments
}

\section{Ksanfomality", AS Selivanov and Yu M Gektin}

\section{Space Research Institute of the Russian Academy of Sciences, Russia}

\begin{abstract}
On March 1 and 5, 1982, experiments in television photography instrumented by the landers VENERA-13 and VENERA-14, yielded 37 panoramas (or their fragments) of the Venus surface at the landing site, gathered for 2 hours of the mission done at the temperature $460{ }^{\circ} \mathrm{C}$ and pressure $9.2 \mathrm{MPa}$. Over the past 35 years, no similar missions have been sent to Venus. Unique archive data of the VENERA spacecrafts missions in 1975 and 1982 are reprocessed with up-to-date techniques, which substantially improved their level of detail. There are a dozen of detected and identified hypothetical living objects found by new analysis of the VENERA television images. Numerous objects exhibiting a complex regular structure and presumably very slow motions have been found. The objects are noticeable in size and may testify to the existence of life on the planet with radically different physical settings. It is concluded that, to investigate the hypothetical life of Venus, a new special mission, much more sophisticated than the VENERA missions (1975-1982), should be urgently carried out.
\end{abstract}

Keywords

VENERA missions, Television photography, Extraterrestrial life, Terramorphism

\section{Introduction}

"It is always dark, dusty, stuffy and windy on the surface of Venus". So wrote V. Sharonov, prof. of astronomy of the St. Petersburg University in his book "Planet Venus" [1], published in 1965. Two years later, in 1967, the first Venus-reached spacecraft, VENERA-4, was crushed by the enormous pressure when descended into the planet's atmosphere. It took a few more VENERA landers to finally establish [2] that Venus is light in the daytime, although the spectrum of illumination differs significantly from the terrestrial spectrum, Figure 1. Additionally, it should be mentioned that duration of day and night on Venus is 58.5 times longer than on the Earth.

Soon after the first publications (2012) on a hypothetical detection of possible life on Venus [3], the unexplained sharp hostility of many researchers appeared to the results of our research that is difficult to explain.

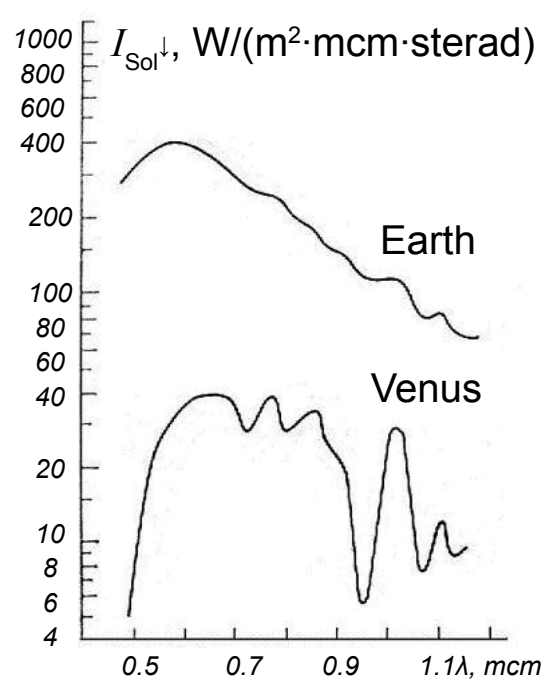

Figure 1: Intensity and spectral distribution of the solar radiation at surfaces of Earth (clean sky) and Venus.

*Corresponding author: LV Ksanfomality, Space Research Institute of the Russian Academy of Sciences, ul. Profsoyuznaya, 84/32, Moscow, 117997, Russia, E-mail: leksanf@gmail.com

Received: August 01, 2017: Accepted: March 20, 2018: Published: March 22, 2018

Copyright: @ 2018 Ksanfomality LV, et al. This is an open-access article distributed under the terms of the Creative Commons Attribution License, which permits unrestricted use, distribution, and reproduction in any medium, provided the original author and source are credited. 


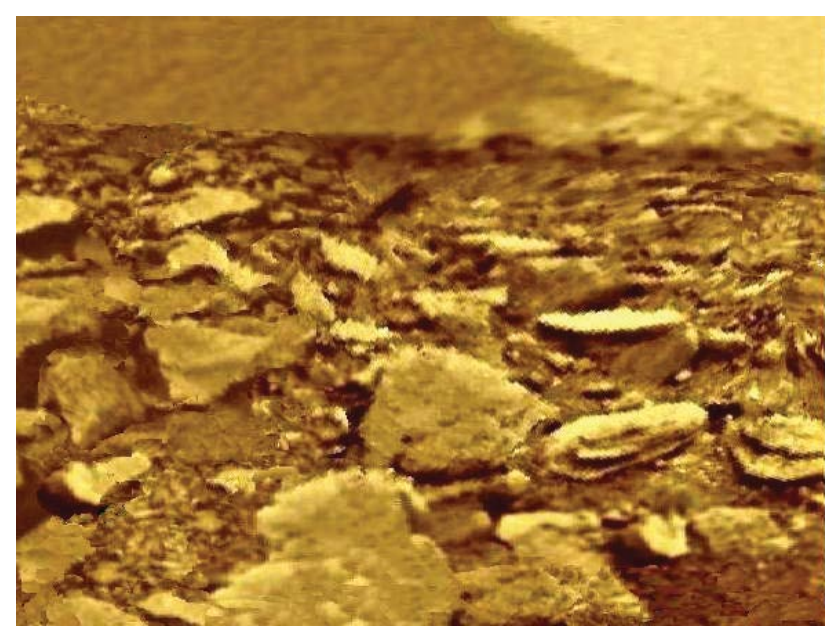

Figure 2: Image of the planet's surface based on VENERA-9 panoramas of the landing site (1975) in modern processing.

If papers written on assumptions, of what kind a life beyond the Earth might be met, are eagerly read and discussed, the experimental data on Venus in the best case are simply ignored. Apparently, is the search for life more important than its find, actually? A number of scholars demanded for "additional evidence". Of course, the additional evidence is needed, however for their obtaining new mission to Venus is needed. More strange is that nobody asked for original material that are in a free access. The hypothetical discovery was made by the study of television images that is the same method, by which Mars is studied now. One can imagine how much extraordinary enthusiasm and noise would appear, if something like that is found on Mars. However, the story is not new. Still in the XIX century philosopher Arthur Schopenhauer (1788-1860) wrote:

"Jede Wahrheit durchläuft drei Phasen:

In der ersten wird sie verlacht,

in der zweiten wird sie wild bekämpft,

und in der dritten wird sie als Selbstverständlichkeit akzeptiert".

\section{TV-Experiment Onboard the VENERA Landers}

There are discussions suggesting that the TV experiments should be described in more detail. Forty two years ago, the first TV images were returned from the surface of Venus. At that time, Soviet investigations of the closest planet carried out with spaceborne instruments were at their height. The VENERA probes performed complex experiments when descending in the atmosphere of the planet. The atmosphere of Venus is so dense that the first module probing the atmosphere, VENERA-4 (1967) was crashed at a pressure of $0.72 \mathrm{MPa}$ at an altitude of around $25 \mathrm{~km}$ above the surface. The next, VENERA-5 and -6 probes (1969) experienced a pressure of $2.7 \mathrm{MPa}$, mea- sured at an altitude of approximately $17 \mathrm{~km}$ and were crushed by the atmosphere, too.

The VENERA-7 (1970) and VENERA-8 probes (1972) were the first to reach the surface in an operating state. The first surface images, "panoramas", were returned later, by the VENERA-9 and -10 spacecraft in fall 1975. Under the atmospheric pressure exceeding Earth's by 92 times and at a temperature of $460{ }^{\circ} \mathrm{C}$, they operated for almost an hour before they stop imaging. Both of landers VENERA-9 - VENERA-10 were equipped with special TV cameras [4-6].

The obtained images were black and white, without many details, but rather sharp (Figure 2). More sophisticated experiments were placed onboard the VENERA-11 and -12 spacecraft in 1978 . To protect the lens windows of the TV cameras during the descent, there were lids covering optical inputs. The lids had to be removed by special pyropatrons after landing. However, the 1978 TV experiment failed: As it turned out, some properties of the Venusian environment were not taken into account at the design of the landers, and the lids were not detached. For more than 1 hour, the cameras returned to Earth images of the inner surface of the lids. The view of the surface at the landing sites remained unknown. Later, it was reported that there were also problems on the VENERA-9 and -10 landers (1975): The lids were detached only on one of the two cameras on each of the landers.

A genuine triumph of researchers were the VENERA-13 and -14 landers (March 1982) [6,7]. The detailed color panoramic images of the surface of Venus (Figure 3), transmitted by the landers, were published by media all over the world. The optical-mechanical TV cameras of the landers $[4,6]$ were designed by a team of specialists from the Russian Space Systems Institute led by coauthors of this paper, Dr. Selivanov and Dr. Gektin. By the start of the work on TV cameras for the VENERA landers, Selivanov's team had already designed the TV-cameras for the LUNOKHOD rovers and Mars landers.

The TV-cameras themselves were unusual in design [4-6]. At that time, arrays of semiconductor detectors of images, CCDs (charge-coupled devices), which are currently used in electronic photographic cameras, had not been developed yet. In the 1960s-1970s, the Lunar and Martian spacecrafts were equipped with film cameras containing automatic devices for chemical developing, electronic scanning, and transmission of the obtained images by radio link to Earth. However, in the TV cameras of the VENERA landers, where the temperature was continuously increasing, it was impossible to use a photo process. The use of ordinary TV camera tubes, unsuitable for operation under high temperatures, was not only more risky, it required significant volume of 


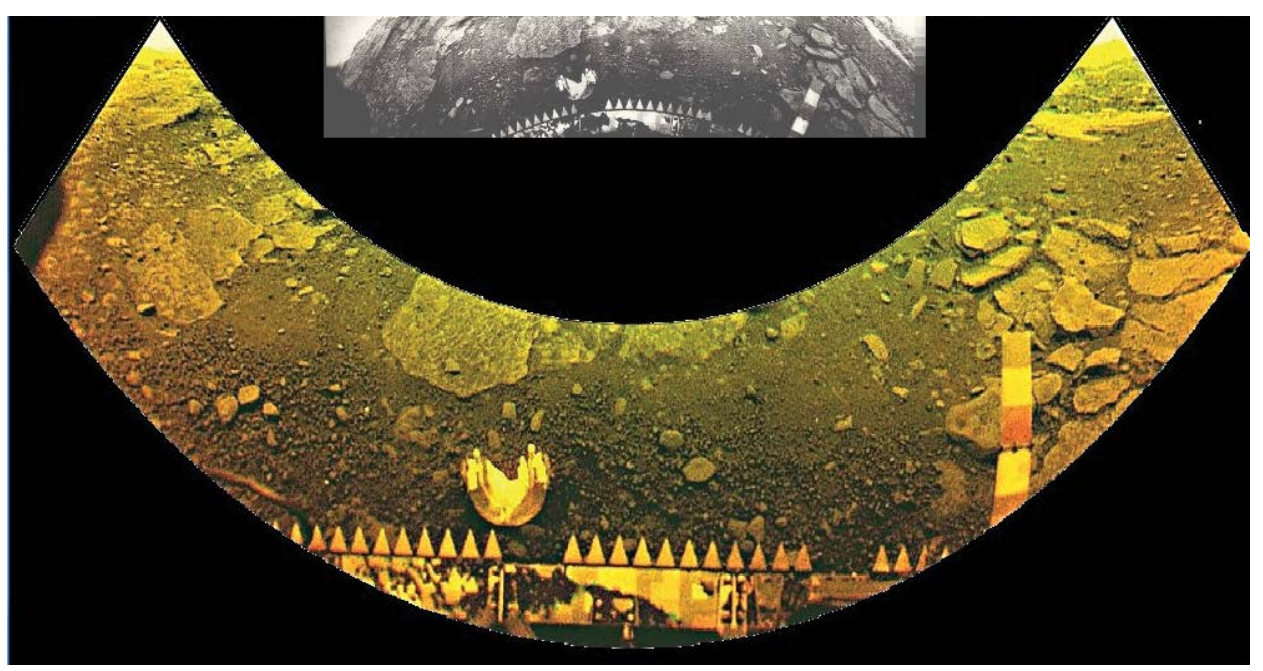

Figure 3: VENERA-13 panorama taken by camera 2 after processing with up-to-date techniques. Geometric distortions have been corrected. Inserted at the top is the same panorama taken from previous publications.

(a)

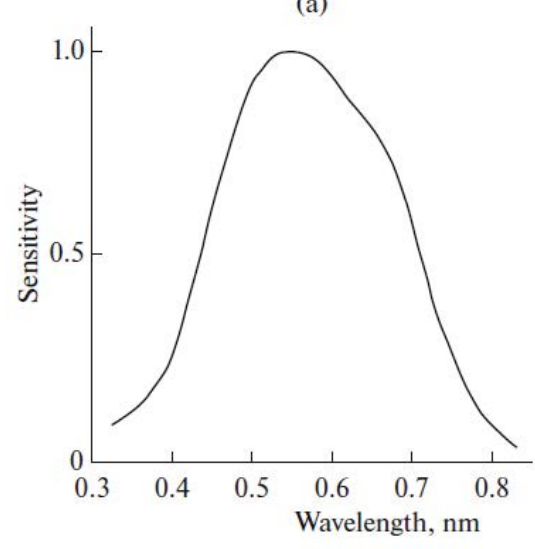

(b)

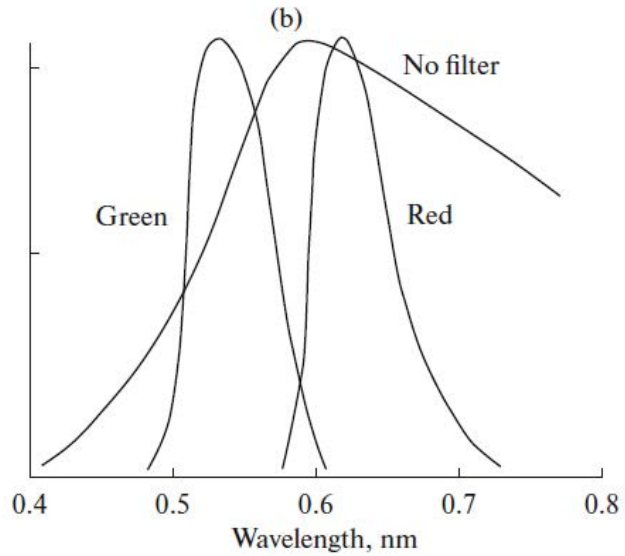

Figure 4: Spectral characteristics of TV-cameras of VENERA-9 and -10 (a) and VENERA-13 and -14 (b) landers.

onboard memory. So it was decided to use a one-channel electronic device, a FEU-114 photomultiplier. The spectral characteristic of the photomultiplier's photocathode corresponds to the multialkali type (Figure 4a); the wavelengths at the 0.2 level of the maximum (at $550 \mathrm{~nm}$ ) are 400 and $760 \mathrm{~nm}$. In front of the photomultiplier's cathode, there was the opaque screen with a diaphragm. The lens formed an image on the screen, and an image element that entered the diaphragm was transmitted as a pixel signal. The aperture size was 21 arc min in the 1975 missions and 11 arc min in the 1982 missions cameras. Thus, the resolution of the panoramas of Venus cedes to normal human vision by 22 times.

It should be mentioned that a direct return of the scientific data from Venus surface to Earth was not impossible, rather it would be useless due to the low signal-to-noise ratio. Instead VENERA satellites were used as re-translators. One full image of the VENERA-13 and -14, a "panorama", extending from horizon to horizon, took 13 minutes to be completed and transmitted. For
2 hours of active functioning the TV-cameras duplicate images up to eight times. The clarity of a picture element that has a fixed size depends on the distance. The (vertical) line resolution was 211 pixels (and 11' arc min); thus, a pixel size of $0.5 \mathrm{~cm}$ corresponds to the distance $0.005 /$ $(11 / 3438)=1.56 \mathrm{~m} \mathrm{(3438}$ - the number of minutes in one radian). Unfortunately, upon heating, the equipment's adjustment deteriorated, and the actual resolution became worse. If the image of a specific object is not single then batch processing and stacking can be used to study the details. A treatment of a single low-noise image can increase its clarity substantially.

Cross-section of the VENERA-9 TV camera (a) and its optical scheme (b) are presented in Figure 5. At the top of the periscope, the scanning mirror had a temperature of about $475^{\circ} \mathrm{C}$, the lower part temperature did not exceed $50^{\circ} \mathrm{C}$. The heat absorber 8 was based on lithium salts. It filled all the free space in the camera and absorbed the penetrating heat due to the phase transition of the material at a temperature of about $25^{\circ} \mathrm{C}$. The me- 

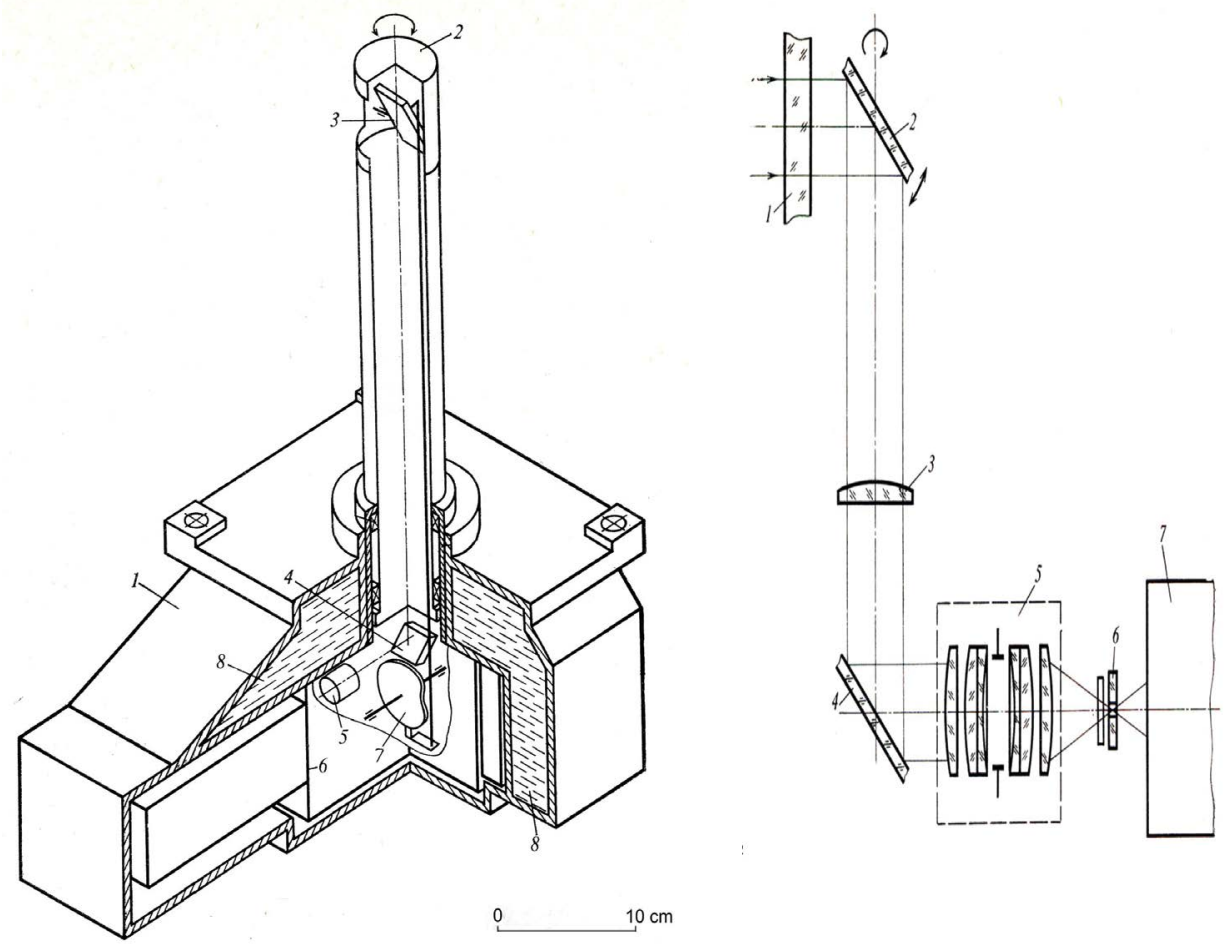

Figure 5: Cross-section of the VENERA-9 TV camera (a) an its optics scheme (b). a) 1-body, 2-periscope, 3-scanning and pivoting mirror, 4-periscope mirror, 5-lens and diaphragm (21 arc min), 6-photomultiplier and electronics, 7-mechanical scanning system, 8-Li heat accumulator; (b) 1-cylindrical quartz window, 2-scanning and pivoting mirror, 3-cylindrical lens, 4-periscope mirror, 5, 6-lens and diaphragm, 7-photomultiplier. The mass of the camera was $5.8 \mathrm{~kg}$ including $2.1 \mathrm{~kg}$ of lithium salts. The camera used $5 \mathrm{~W}$ power.

chanical scanning system transmitted the sawtooth motion to the scanning mirror through a thin cable of 250 $\mathrm{mm}$ length. The reverse time of the scanning was used to calibrate the instrument and include the housekeeping data in the transmitted line. In electronics, an automatic gain control system was applied through negative feedback acting on the high voltage on the photomultiplier. A curious feature of the experiment was the installation on each VENERA-9 and -10 landers two powerful (100 $\mathrm{W})$ spotlight illuminating the surface. Since the suggestion of constant darkness on the surface of Venus could not be ignored, the use of spotlight guaranteed illumination up to 4000 lux. Later, the effect of increasing the natural illumination using spotlight was hardly detected in the recorded video signal.

The area surrounding the landing site was reflected by the scanning and pivoting mirror 3 (Figure 5a) mounted on the top of the periscope, and the mirror swung within an angle of approximately $40^{\circ}$. In the VENERA-13 and -14 and VENERA-9 and -10 cameras, one sweep of the mirror took 0.78 and $3.5 \mathrm{~s}$, respectively. In such a way, one picture line whose elements passed by turns through the diaphragm, was formed. In contrast to a usual TV, lines were vertical; at the end of each sweep, a special mechanism rotated the mirror axis by an angle corresponding to the aperture size, in the plane perpendicular to the sweeping direction and a next line of the picture was formed. In the VENERA-13 and - 14 cameras, a whole image contained 1000 lines, while a line itself contained 211 elements of the image (pixels) and 41 elements of the service information. (The image transmitted by the VENERA-13 lander and processed with up-to-date methods is shown in Figure 3). To protect the camera from thermal radiation, a periscope system was used that placed the camera at the lower end and the scanning mirror at the top. The VENERA-13 and -14 cameras transmitted split-color images obtained with red, green, and blue filters (Figure 4b) (rather than only black and white of the VENERA-9 and -10 cameras). However, since the radiation in the blue spectral range is almost completely absorbed by the atmosphere, the blue panoramas were worthless and were not used. To scan a whole image took $13 \mathrm{~min}$.

Though the guaranteed operation time of the VEN$E R A-13$ and -14 cameras was 30 min they worked successfully for more than $2 \mathrm{~h}$ and transmitted a considerable number of panoramas and their fragments. The images and service information were corded in a 10-bit system (1024 levels in total, where 512 levels were for the image) and transmitted via the omnidirectional antenna of the lander to the orbiter. Each of the landers was equiped with two cameras mounted on its opposite sides. 


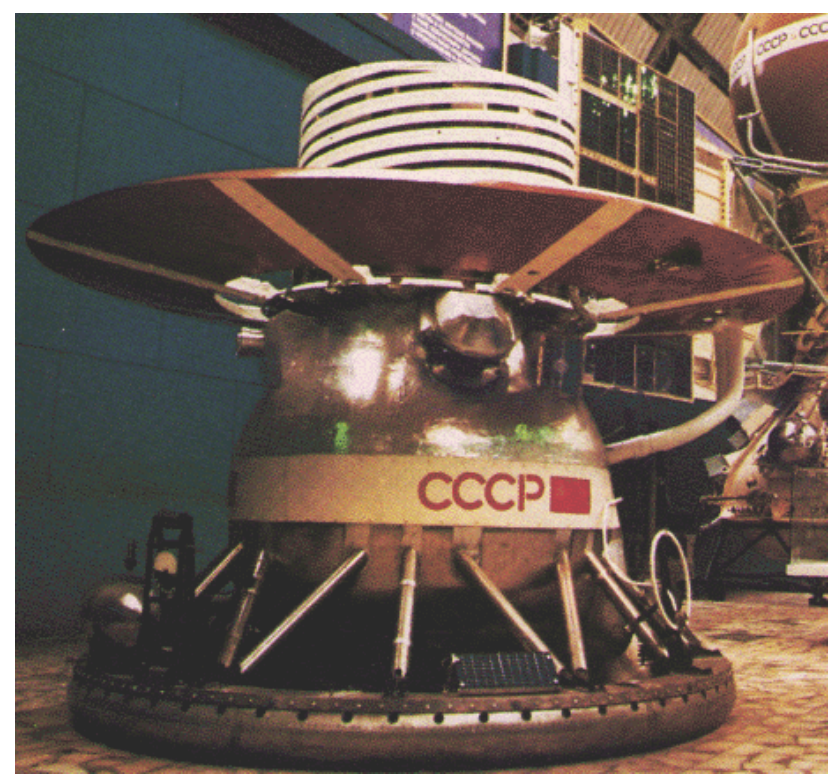

Figure 6: VENERA-13 lander at laboratory tests. The TV-camera input window is placed in the center of the image, just below the parachute disk and is covered by the lid.

The resolution in the VENERA-9 and -10 panoramas was almost two times worse than that of the VENERA-13 and -14 cameras. Their cameras produced only black and white pictures. The 180-degree panorama contained 517 vertical lines of 115 pixels each, and it took approximately 30 min to obtain a complete image. For the VENERA-9 and -10 the angular resolution (corresponding to a single pixel) was 21' [4]. The input window of the optical system was placed $82 \mathrm{~cm}$ above the landing buffer of the VENERA-9 and -10 (and $90 \mathrm{~cm}$, VENERA-13 and -14 , Figure 6) landers.

The VENERA-9 and -10 landers worked on the surface of the planet for 50 and $44.5 \mathrm{~min}$, respectively. The VENERA-9 panorama actually covered $174^{\circ}$ and the duration of imaging (with it's real-time transmission) was $29.3 \mathrm{~min}$. After that, the right portion of the panorama, within $124^{\circ}$, was taken again. The coverage of the VENERA-10 panorama was $184^{\circ}$; two fragments at the beginning and the end of the image, by $63^{\circ}$ and $17^{\circ}$, were subsequently repeated. The image was corded in a 6-bit system (64 levels), transmitted via the omnidirectional antenna of the lander to the orbiter (in a 48-h orbit), and relayed in real time to the Earth by its narrow beam antenna. A fragment of the VENERA-9 panorama is shown in Figure 2.

\section{The VENERA Panoramas: Hypothetical Flora and Fauna}

For thousands of years, humanity has wondered whether there is life outside the Earth. Experiments onboard the VENERA landers were designed to gain general ideas about the surface of the planet. It did not occur to anybody at that time to search for life on a planet with an anoxic carbon dioxide atmosphere having a pressure of $9.2 \mathrm{MPa}$, and a temperature of $735 \mathrm{~K}$ near the surface. However, now it cannot be ruled out that the TV images of the surface of Venus, taken 43 and 36 years ago and reprocessed with up-to-date software, evidence the presence of flora and fauna on this planet, strange as this may seem. Since experimental data on the supposed habitability of Venus were obtained for the first time, it was impossible to count on the support of specialists.

Subconsciously, all positions of critics have been based on variations of the statement: only the Earth's conditions are suitable for life. "We are the best and all our physical conditions are the best too". Based on this idea, limited "habitable zones" are drawn in schemes of extrasolar planet systems and are under the study of theoreticians. Nevertheless, after the very first publication that was devoted to the discovery of hypothetical life on Venus, one of the world leading biophysicists, academician A. S. Spirin, commented as follows: "In addition to demonstrating the extremely unusual "strange stones" discovered on Venus, that, generally speaking, baffle the attempts of any purely "mineralogical" interpretation of the images obtained and their independent movement, the article seems to be very important in another aspect. It begins the discussion about the principal possibility of the existence of large living, independently moving objects, under very extreme temperature, pressure and illumination conditions... I have found nothing that would principally contradict the possibility that the living organisms exist under Venusian conditions..." Of course, comparing them with the type of life on Earth should be another type of biophysics.

As for the criticism, together with the "Earth chauvinism", there is another, perhaps political, item: NASA has spent much money for so many missions to Mars. No Earth-like type of life was found. (The very special question is - why?). Was there an alternative that was forgotten by 'those who made the decision'? One can understand their feelings if triggered by the news on hypothetical life found on Venus.

The problem of hypothetical Venusian life appears out of the "shadow of doubt" already. One of the authors of the VENERA TV experiments and co-authors of this paper, Dr. Gektin, concluded: We don't like the idea of life on Venus. However, we are not able to propose any other explanation to what we see in the VENERA panoramas.

The VENERA TV images were re-processed using modern processing techniques. There are entities that one can consider to be signs of hypothetical life on hot waterless Venus, regardless of how crazy this assumption sounds. The pictures revealed a dozen previously undetected strange objects that can attest to the fact that Ve- 


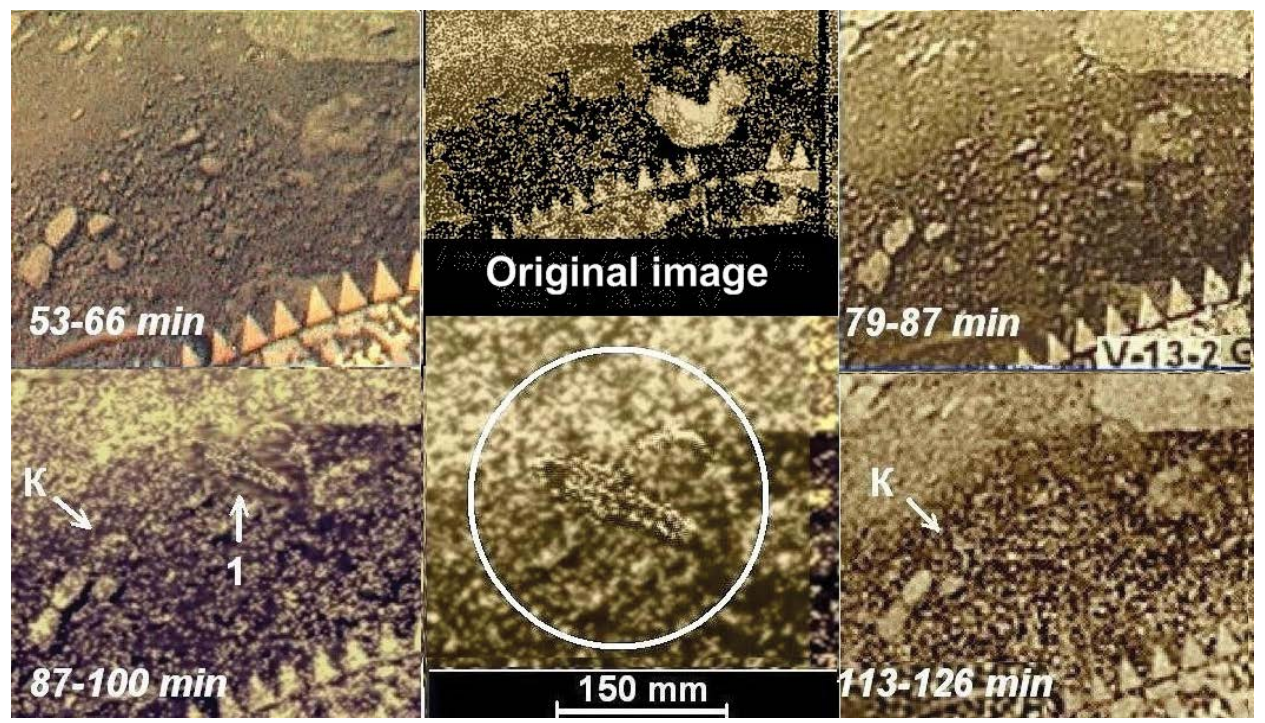

Figure 7: The object "scorpion" appeared in the panorama $6 \mathrm{BW}$ obtained from the $87^{\text {th }}$ to the $100^{\text {th }}$ minutes. In images obtained before the $87^{\text {th }}$ and after the $113^{\text {th }}$ min, the object is absent. In the images of minutes 87-100 and 113-126 min, to the left, in the group of stones, there emerged a new object $\mathrm{K}$ with a changing shape. The central part of the figure, at the bottom, shows the result of the "scorpion" image processing and its size.

nus does possess life. For the moment, it is impossible to prove that the objects are alive in fact because they cannot be touched. However, the opposite is true also, that nobody can place errors into the processing of the images. Instead, critical arguments boil down to the famous humorous statement of A.P. Chekhov, in his 'Letter to my neighbor-scientist': "this cannot be, because it never can be".

Looking for signs of life beyond the Earth, researchers usually proceed from the physical settings that the Earth possesses. In addition, it is assumed that the hypothetically detected life will not be too much different from ours. Because we are the best, and our planet is the best. Earthly chauvinism. And our physical settings are the best, too. Thus, in essence, we are looking for ourselves. If one looks for habitability, then it is obligatory a planet whose atmosphere contains oxygen, a sign of life. Although experts argue that the first 2 billion years earthly life existed well without atmospheric oxygen. And yet the temperature at the surface of the planet should only be, as is comfortable for us. (Well, geochemists remind us that at high temperature, reactions are accelerated considerably. Even compounds are produced which do not arise under normal conditions. And a significant part of exoplanets has high temperature at their surface).

Nevertheless, many researchers believe that the search for life outside the Earth should be extended to completely different physical conditions. The well-known biophysicist A.Azimov, considering the chemistry of life existing at higher temperatures, called nucleic acids and proteins based on nitrogen, rather than carbon. In the book "Life in the Solar System and Beyond". B.Jones writes: "Our approaches should not be built too literally on the variant of life that exists on Earth, i.e. based on RNA, DNA (carbon and liquid water) and a specific set of proteins ... Following some search options, we would be able to detect life based on a completely different chemical composition (without carbon and water)". The leading Russian biophysicist A.Spirin, considering first reports on the discovery of a hypothetical life on Venus, at temperatures of $460{ }^{\circ} \mathrm{C}$, notes: "Being a molecular biologist,... I have found nothing that would principally contradict the possibility that the living organisms exist under Venusian settings".

\section{Fauna of Venus}

Due to the availability of many duplicates of the images obtained and their low level of masking noise, the VENERA panoramas permit reliable identifying and exploring some new types of hypothetical life forms of Venus. At the time of this writing, 5 years have elapsed since the submission of the first manuscript for publication that was devoted to hypothetical signs (Figure 7) of life on the planet Venus. An accumulation of new objects increased gradually. These objects hypothetically have characteristics of living creatures - fauna and flora. At the initial stage of the investigation few relatively large objects were found, nicknamed as "scorpion", "hespera", "owl", "bear-cub" and "snake" (Figure 8).

The first detected interesting object, conditionally called "the scorpion", was discovered in the panorama number $6 \mathrm{BW}$, near the $90^{\text {th }}$ minute of scanning. Before that, the instrument had already been working for 1 hour and 27 minutes (moment of the start of scanning the $6 \mathrm{BW}$ image); therefore, our first suggestion was that this regular structure is a product of destruction of some 


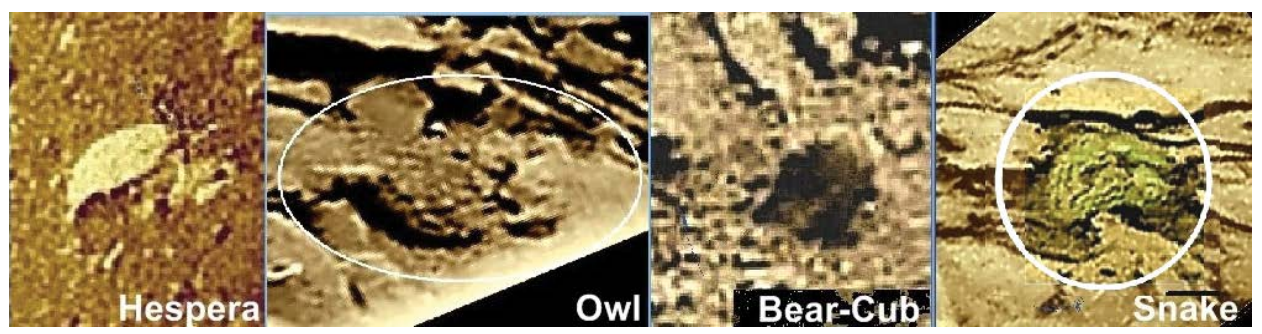

Figure 8: Fauna of Venus. Gallery of some entities described in published papers. All their names are conditional.

part of the lander itself. But the lander VENERA-13 continued to work for almost a whole hour after that, indicating that there could not be any destruction, otherwise all instruments would have failed due to catastrophic overheating immediately. An analysis of the technical documents showed that all external operations (such as throwing the lids away, operation of the drilling setup) terminated in less than 30 minutes. The assumption of a split detail is also in conflict with the fact that the object is absent in the subsequent images.

The history of the emergence and disappearance of the "scorpion" is illustrated in Figure 7. In images obtained before the $87^{\text {th }}$ minute, the object is absent. The object is absent as well in the later image 6G (113 to $126 \mathrm{~min}$ ). A likely cause may be in that, if the object moved, then, as it went away to a distance of 3 to 4 meters, it became indistinguishable from stones. At such a distance, as a minimum, the object should have moved away in 26 minutes, the time up to the next panorama 6G in Figure 7.

In the course of scanning, the image of a moving object can be distorted. The image fragment with the "scorpion" was obtained during 32 seconds. The object motion could cause, for instance, its seeming lengthening or shortening in the image. However, later it was found that all hypothetical living objects that show movements, are very sluggish. Their speed doesn't exceed $1 \mathrm{~mm} / \mathrm{s}$.

The "scorpion" has a length of 15 to $17 \mathrm{~cm}$ and a complicated structure resembling some terrestrial arachnida or insects. (One may remember that these categories do not belong to fauna). In its immediate vicinity in the panorama $6 \mathrm{BW}$, there emerged a formation like a half ring of the same size. It is a moving object of another class, which does not disappear but takes another position in each image. The complex and regular shape of the "scorpion" cannot be a result of a random combination of light, half-tone, and dark points. The image of the "scorpion" consists of 940 points, while the number of points in the whole panorama, covering $177^{\circ}$, is $2.08 \cdot 10^{5}$. The probability $\mathrm{p}$ of forming such an image, if we count only the number of combinations, is vanishingly small and is in fact excluded. In addition, there is a physical indication of "scorpion" reality: An analysis reveals a shadow under the object. Shadows certainly cannot form near a random combination of points. A shadow shows that the object has a relief and is located over the surface. Most probably, the emergence and then disappearance of the "scorpion" are connected with destruction and lateral ejection of soil in the course of landing rather than a direct influence of the wind [8]. The vertical speed of the spacecraft at landing, found by a dynamical method [9], was $7.6 \mathrm{~m} / \mathrm{s}$, while the lateral speed was approximately the same as that of the wind $(0.3$ to $0.5 \mathrm{~m} / \mathrm{s})$. The stroke amounted to $50 \mathrm{~g}$ of Venus. The lander destroyed the soil to a depth of about $5 \mathrm{~cm}$, threw it aside, and the soil could have covered the "scorpion". The place where the "scorpion" appeared was studied in the whole sequence of panoramas, from the $7^{\text {th }}$ to the $119^{\text {th }}$ minute. At first, a shallow ditch of about $100 \mathrm{~mm}$ long is seen on the soil thrown out. Then the sides of the gutter are lifted, and its length grows to about $150 \mathrm{~mm}$. The gutter orientation is the same as that of the "scorpion". In an hour, the regular structure of the "scorpion" emerged from the ditch. At the $93^{\text {rd }}$ minute, the "scorpion" probably completely got out of the soil that had covered it, whose whole layer was not thicker than 1 to $2 \mathrm{~cm}$. At the $119^{\text {th }}$ minute, it had already gone. Thus, the object needed about an hour and a half for the "rescue" operation. This apparently points to its restricted physical abilities.

As another cause of the object emergence and subsequent disappearance, the possible role of the wind was also considered. At the measured wind velocity of 0.48 $\mathrm{m} / \mathrm{s}$ at the landing point [8] and the atmosphere density of $60 \mathrm{~kg} / \mathrm{m}^{3}$, the wind "head" pressure $\rho v^{2} / 2$ on the "scorpion" cross section gives a pressure force of about $0.08 \mathrm{~N}$, which is insufficient for its displacement.

The coincidence of the scanning time of the panorama $6 \mathrm{BW}$ with the emergence of the "scorpion" object was a great fortune of the experiment. Lucky was also the very position of the survey where the resolution made it possible to follow both the development of the events described and the disappearance of the object in the last panorama.

When experience using image processing was accumulated, the VENERA-14 panorama allowed an approach to the finer details, similar to the Figure 3. An important role was played by additional image processing, 
image geometric correction and the presence of up to eight duplicates of images that were obtained with good quality and low levels of noise. This arrangement enabled the selection and staking of images and their fragments. As a result, we managed to find and learn few new types of hypothetical inhabitants of the planet at the VENERA-14 landing site: "stems", "amisadas" and a "snake".

Some of other hypothetical living objects, static or showing movements, are placed in Figure 8.

"Hespera" reminds of felt leaves by its shape, however it is lifted above the surface. There are other similar objects. They were conditionally called hesperos, which

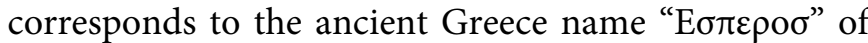
the planet Venus. A large hesperos is shown in Figure 8. It is seen at an angle of $35^{\circ}$ to the horizon. One may distinguish the transverse band in the middle of the object. Under the hesperos, the shadow is seen, which indicates that the hesperos is elevated above the surface. The sizes of hesperos vary but, basically, are close to 20-25 $\mathrm{cm}$ including the "tail", as is the case in Figure 8. Similar objects were discovered both on opposite sides of the lander and on the panorama of the lander VENERA-9, at the distance of $4000 \mathrm{~km}$ away. During the time of the mission, both objects were scanned multiple times; however, no displacements were found. The right-hand side of the object is terminated by projecting prolate forms that resemble whiskers intrinsic to numerous living species of the Earth.

The object "Owl", first called "a strange stone with a white tail" found in the place of the VENERA-9 landing. Its size is about a half meter. The first assumption of the object as an inhabitant of Venus was made in 1975. The complex symmetric form (with respect to its longitudinal axis) of the "owl", with a long white "tail" on its left, makes it stand out against the background of the rocky surface of the planet at the VENERA-9 landing site.

Terramorphism intrinsic to certain types of possible Venusian fauna, apparently, relates to the deepest puzzles of searching for life in the Universe. This object "BearCub" was found in 2012 in the VENERA-9 panorama (obtained back in 1975). In contrast to the sharp contours of the surrounding stones, the surface of the object is rather "soft" and even may be called "fluffy". The object is seen almost from above at an angle of $62^{\circ}$ to the horizon. BearCub seems to rest on its extremities with a certain clearance between them. The extremities resemble animal paws. According to the positions of shadows cast by BearCub and the surrounding stones, one may assume that its height and length (width) being 32 to 34 $\mathrm{cm}$ and about $16 \mathrm{~cm}$, respectively.

The greenish coiled "snake" about $40 \mathrm{~cm}$ long is marked in Figure 8 by a white circle. Color of the Figure is exaggerated. The object actually resembles a convolved snake. The surface of the object is covered by regularly located spotted cells. The object is positioned in a small ( 5 to $10 \mathrm{~cm}$ ) depression. The object exhibits certain terramorphic features intrinsic to Earth reptiles, e.g., the cellular structure of its surface, in itself, or of its color. The snake demonstrares small displacements of the body and changes in its positions with respect to other parts of the object or to stone plates, which exceeds displacement had been found for other objects. For example, the displacement of the crest on snake's neck in sequential frames for $30 \mathrm{~min}$ attained 3 to $4 \mathrm{~cm}$.

The interest in the hypothetical autotrophic flora of the planet as a source of the existence of its fauna was noted in published scientific papers. It is natural to assume that, like on the Earth, the Venusian fauna is heterotrophic, and the source of its existence is hypothetical autotrophic flora (produced in unknown type of photosynthesis). Although the direct rays of the Sun, as a rule, do not reach the surface of the planet, there is enough light for photosynthesis of the Earth-like known type there. In the case of the Earth, a diffuse illumination of 0.5-5 kLux is sufficient for photosynthesis even in the depths of the dense forests. The measured luminance on Venus is of the same order, at the range of 0.5 to $9 \mathrm{kLux}$, having very special spectral composition (Figure 1). Of course, photosynthesis at high temperatures $\left(460{ }^{\circ} \mathrm{C}\right)$ and in a non-oxidizing waterless environment should be based on a completely different, unknown biophysical mechanism.

\section{Flora}

The first stem object was detected due to its being close to the entrance of the TV-camera, and others were detected by similarities in their shapes and positions to the first "stem". It has a large bulge at the top end, a "burgeon", with a lighter center. (Shown below are other stems where the "burgeon" is observed developing to a flower). In input images, a "stem" resembles only a thin scratch, but it is repeated at all panoramas and in the same place (Figure 9). When processed, the "scratches" are almost vertically arranged thin knotty trunks, which are $0.3-3 \mathrm{~cm}$ thick and $0.2-0.5 \mathrm{~m}$ (and maybe more) tall. At the base of the 'stems', there are features that resemble leaves in a quatrefoil. On panoramas, they look grey or light. Each of the "leaves" has a size of approximately 5-10. In the vicinity of VENERA-14, there other, more distant stems.

The "stem" (Figure 10) is located close to the camera. Each of the "leaves", possibly, has a radial structure (Figure 10). All of the "stems" found are placed vertically.

To find the height $z$ of the stem in Figure 10, one should use geometric relations and a photoplan (because, on the original panoramas, the distances are significantly distorted). The input window of the TV camera is located 

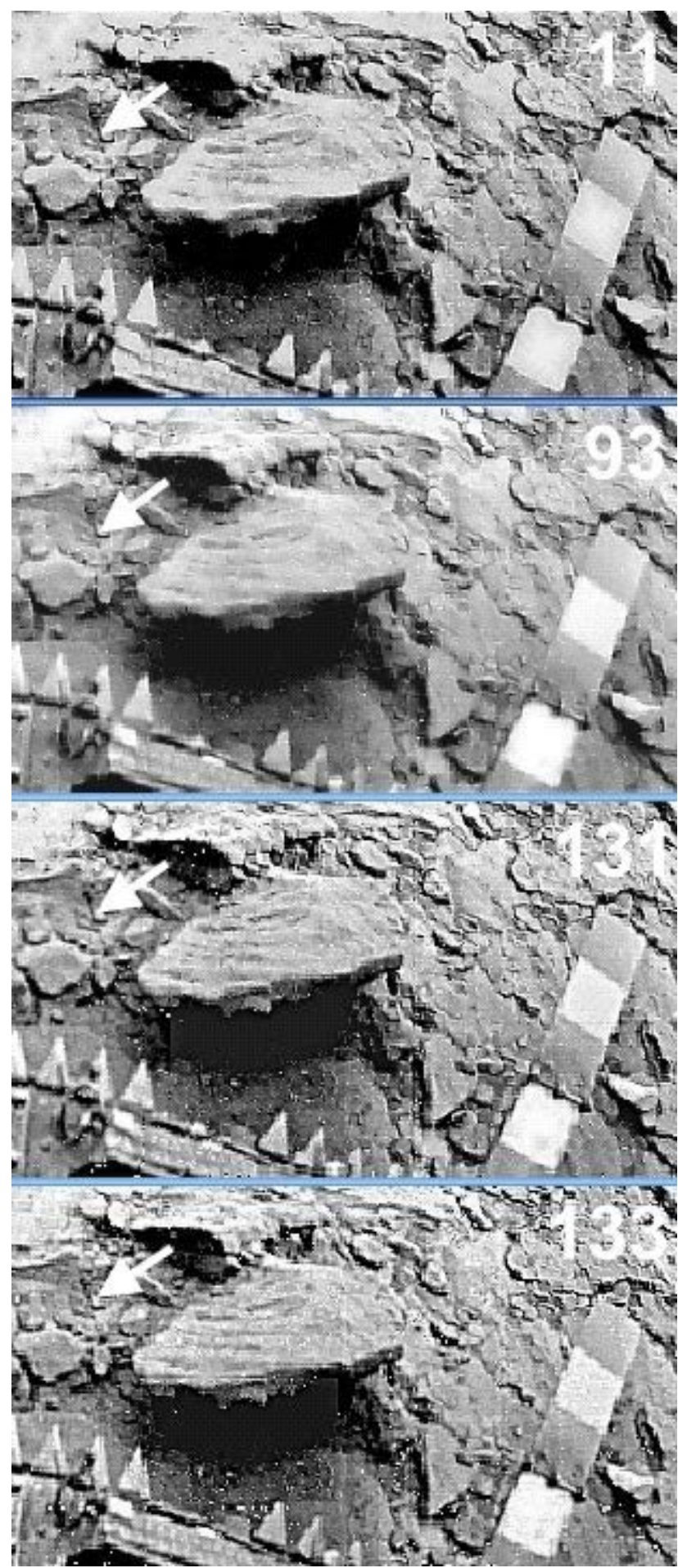

Figure 9: 4 repeated fragments of the surface image at the landing site of VENERA-14. A "scratch" is shown by arrows.

at a height of $90 \mathrm{~cm}$, and from the distance and the right triangle, the stem height is $z=42 \mathrm{~cm}$. An error is possible as the ground surface is uneven.

Another similar "stem" was found in VENERA-13 panorama, whose landing site was about $900 \mathrm{~km}$ away from VENERA-14. The "stem" (Figure 11) is lower than one in Figure 10; it is more distant, and the stem itself is not easy to notice, although there are eight distinct images (duplicates), which allows for batch processing.

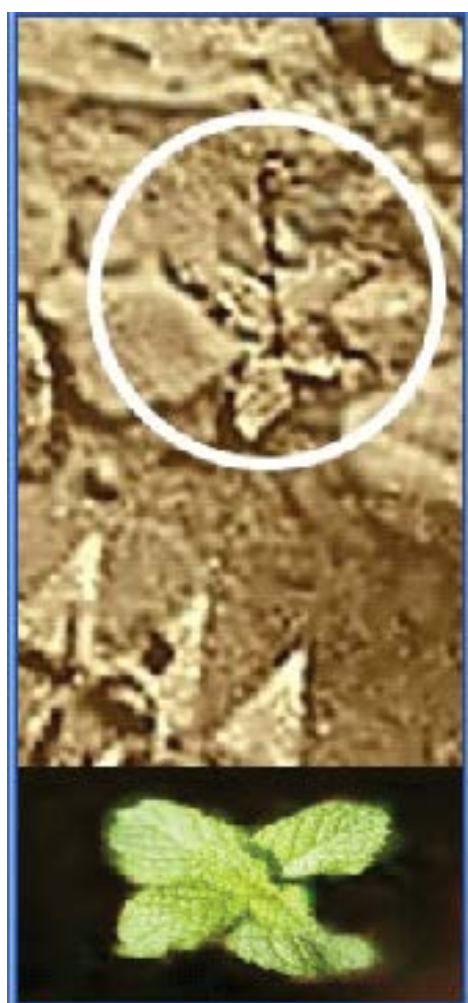

Figure 10: The first found object of the "stem" type is a thin vertically arranged knotty trunk that has a height of approximately $40 \mathrm{~cm}$ and a thickening ("burgeon") on the top. At its base, on the surface, there is a group of details that resembles a quatrefoil. The "stem" is located at a distance of approximately $40 \mathrm{~cm}$ from the landing buffer of the VEN$E R A-14$ lander. It is seen from above. Bottom: A sample of an earthly cruciferous plant.

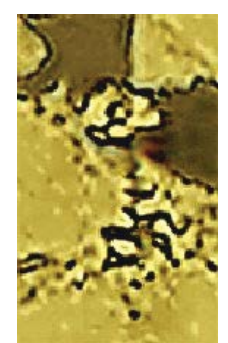

Figure 11: "Flower" - the object in VENERA-13 panorama with lowered contrast and detailed image of the "flower", its light central part and leaves at the base. The diameter of the flower and the "quatrefoil" at its base are $6-8 \mathrm{~cm}$.

The attention is drawn to the top of the stem, which appears as a triad of bright dots that are visible on all of the original high-contrast images. The position of the triad is not identical in successive frames. It varies slightly with respect to the adjacent light-colored stone on top of it. As was shown by the animation, changes arise from the swinging of the triad by the wind. The wind speed is low, about $0.4 \mathrm{~m} / \mathrm{s}$, but the gas density is high.

It has been suggested that the complex structure of the top of the "stem" is an opened burgeon. When processing the image with a decreasing contrast, this assumption was 
confirmed and allowed us to see the whole "flower", of a regular shape (Figure 11) and with a white spot (pestle?) in the center and the surrounding petals. The top of the "stem" is more complex than the bud in Figure 10. The object is visible from above, and its height, which is found by its position on the photoplan, is only approximately $20-30 \mathrm{~cm}$ at the base in the crack between the stones. At its base, there is a group of four bright details, similar to the "quatrefoil" leaves shown in Figure 10. The flower is composed of six to eight light petals. The "flower" size is approximately the same as a "quatrefoil" at the base of the "stem". The VENERA-13 panorama has been organized in such a way that Figure 11 represents only a fragment of the black-and-white image; thus, one can talk about only the bright colors of the petals, and their color in Figure 11 is unknown.
Another interesting small bright quatrefoil was detected at the center of the VENERA-14 panoramas in a depression, close to the landing buffer (Figure 12.1). In contrast to Figure 10, its "leaves" are very bright, only slightly darker than the buffer. One of the quatrefoil elements is in the shadow of a stone. The dimensions of the "leaves" are not more than $2 \mathrm{~cm}$. Despite its smaller size, the object similarity with Figure 10 is obvious. The "stem" itself on the source panoramas is difficult to see; it was isolated by using a gamma-correction and processed. The height of the plant observed from above is approximately $10 \mathrm{~cm}$.

There is a "flower" seen on its top. When the image is processed, the "stem" gets viewed as in Figure 12.2. The

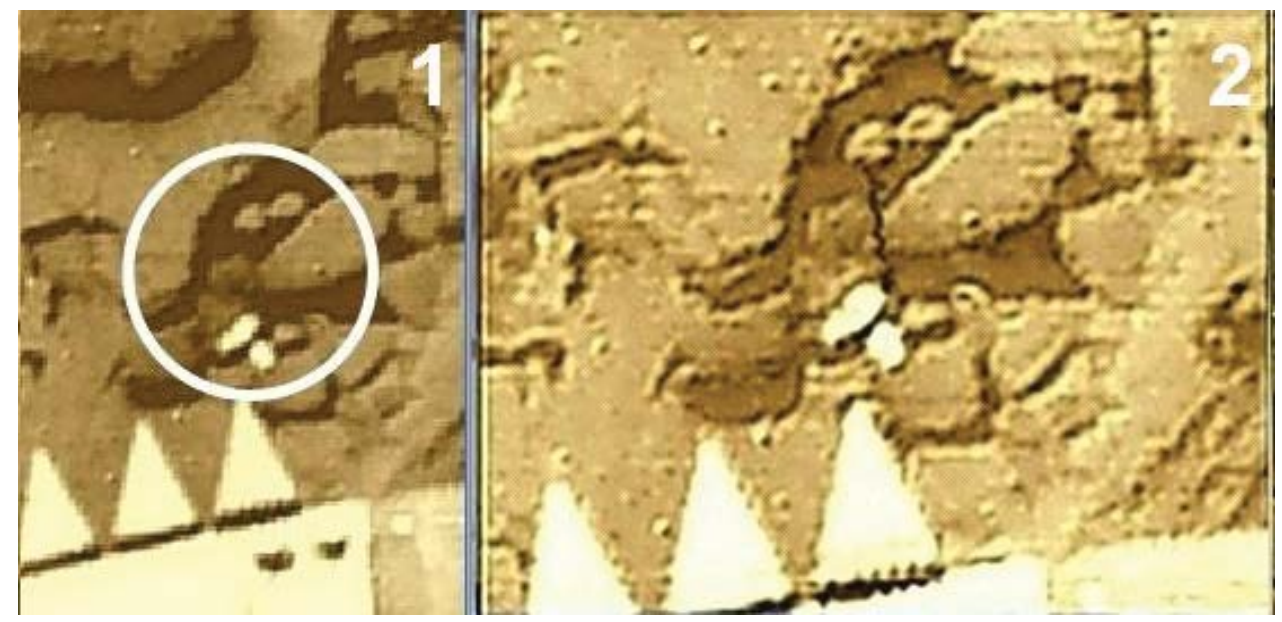

Figure 12: "Stem" with a bright "quatrefoil" (1) located directly at the landing buffer of VENERA-14. The processed image is shown in frame (2). To the right of the "flower", there is another "flower" seen, the "stem" of which is apparently situated behind the stone.

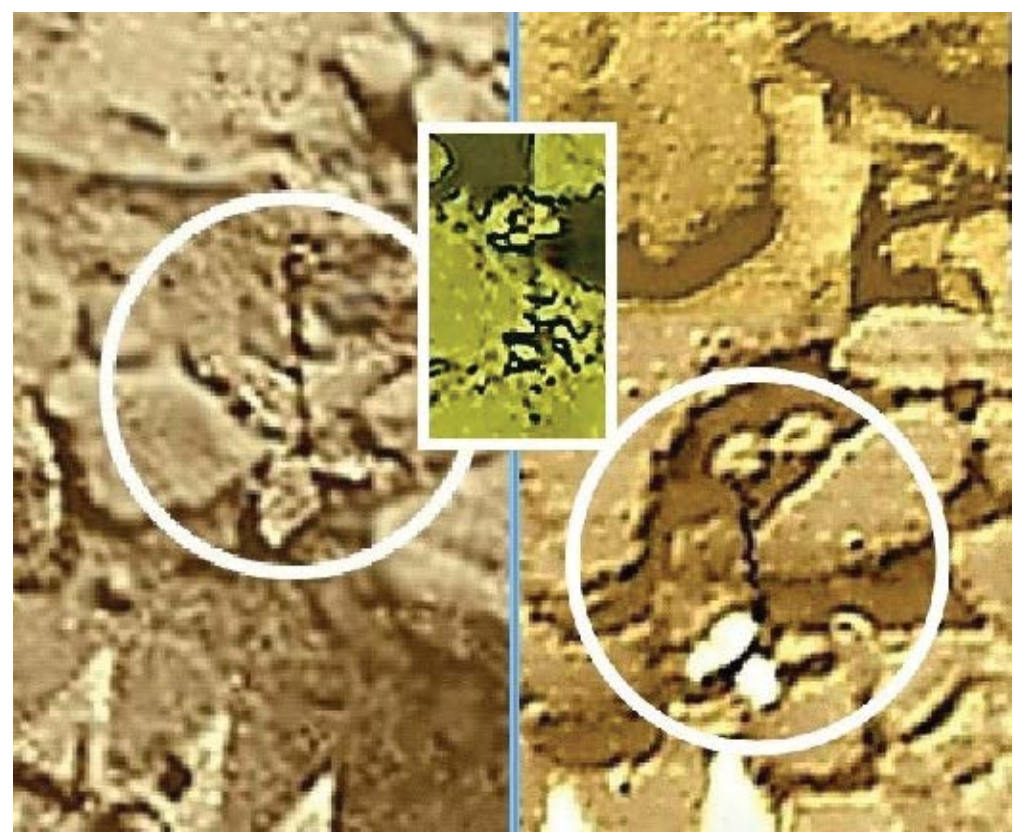

Figure 13: 3 stems with "buds" and "flowers" found at different landing sites. 
dimensions of the "flower" are approximately $2 \mathrm{~cm}$, also. To the right of it, another "flower" is visible, the "stem" of which apparently is placed behind the stone. In Figure 12, "stem" and "flower" are seen against the background of contrasting details and cracks in the stone slab recess. The "stem" rises from the recess. The object is relatively close to the camera (less than $1 \mathrm{~m}$ ), but the "flower" is small, and compared with Figure 10, the resolution is low.

\section{On the Possible Role of Burgeons and Flowers}

The landing site around the landers VENERA-13 and VENERA-14 showed rare vertically oriented objects that were similar to the stems of terrestrial plants. Another similar stem was found in VENERA-9 image. Probably, the 'stems' are widespread on the planet, because they are met at landing distances between the three different VENERA missions sites that were 900 and $4500 \mathrm{~km}$ apart. The both VENERA-14 cameras registered stems at opposite sides of the lander. The VENERA-9 and VENERA-13 cameras registered stem at one side only. The stems are important objects of a hypothetical Venusian flora discussed in $[10,11]$. If the tops of the stems really are burgeons and flowers, one should reflect their role. The flowers of terrestrial plants are intended for their pollination and reproduction. Pollination is conducted either by insects or by the wind. Wind-pollinated plants do not require blooms in principle, for example, the case of the poplar "fluff". Flowers attract insects. Do the tops of the stems in Figure 10, Figure 11 and Figure 12 at least indirectly, hint on the likely participants in the process of pollination?

Terramorphism of hypothetical objects of the flora and fauna of Venus was observed repeatedly in many entities $[10,12,13]$ for example, a terramorphic is object "mushroom", not presented here. Flowers with their petals in Figure 13 are new objects that are surprising to find, and it is surprising to find the occurrence of the same forms of living objects on different planet that have radically different physical settings. However, what are the laws of nature that determine the recurrence of terramorphism hidden in such markedly different environments?

The high-density hypothetical habitability of Venus suggests that its surface is more similar to the bottom of a sea shoal on Earth than on the Earth's surface. If the autotrophic flora is a possible source of energy that could be used by the hypothetical fauna of the planet, then one may think that the detection of "stems" would be corroborated. However, this autotrophic nature itself does not solve the problem of feeding to the fauna by these very rare plants. Smaller vegetation, such as grass or moss, is not resolvable in the VENERA pictures. Although the direct rays of the Sun, as a rule, do not reach the surface of the planet, there is enough light for photosynthesis of the Earth-like type there. In the case of the Earth, a diffuse illumination of 0.5-5 kLux is sufficient for photosynthesis even in the depths of the dense forests. The measured illuminance on Venus is of the same order, at the range of 0.4 to 9 kLux. Of course, photosynthesis at high temperatures and in a non-oxidizing environment should be based on a completely different, unknown biophysical mechanism.

\section{Conclusion}

The VENERA TV-experiments (1975 and 1982) were intended to provide a common understanding of the planet's physics, its surface and atmosphere. But the results obtained are revolutionary. There are a dozen of Venusian hypothetical leaving creatures found in TV results of VENERA missions. We need an urgent implementation of a new mission, to explore the surface of Venus confirming a hypothetical existance of its fauna and flora. The mission should be special and much more complicated than the VENERA missions and provided with new photo electronic technique. Nevertheless, the progress of technical equipment is adequate for it.

\section{References}

1. Sharonov VV (1965) The planet Venus. Moscow, Nauka, Fizmatlit, 256.

2. Moshkin BE, Economov AP, Moroz VI (1983) Spectrophotometric experiment on VENERA-13, -14 landers. Kosmich Issle 21: 236-245.

3. Ksanfomality LV (2012) Possible detection of life on the planet venus. Doklady Physics 57: 367-372.

4. Selivanov AS, Chemodanov VP, Naraeva MK (1976) TV experiment on the venus surface. Kosmich Issled 14: 674677.

5. Keldysh MV (1979) First panoramas of the surface of venus. Moscow, Fizmatlit, 132.

6. Selivanov AS, Gektin Yu M, Gerasimov MA, Nosov BI, Naraeva MK, et al. (1983) Continuation of the TV investigation of venus surface by means of landers. Kosmich Issled 21: 176-182.

7. Ksanfomality LV (1985) Planet Venus. Moscow, Fizmatlit, 376.

8. Ksanfomality LV, Goroshkova NV, Khondirev VK (1983) Wind velocity at the venus surface obtained by means of acoustic measurements. Kosmich Issled 21: 218-224.

9. Baklunov AM, Karyagin VP, Kovtunenko VM (1983) Automatic interplanetary stations VENERA-13, -14. Kosmich Issled 21: 151-153.

10. Ksanfomality LV (2014) Hypothetical flora and fauna of venus. Acta Astronautica 105: 521-533.

11. Ksanfomality LV, AS Selivanov, Yu M Gektin, GA Avanesov (2016) Signs of hypothetical flora on the planet venus: Revision of the tv experiment data (1975-1982). Cosmic Research 54: 217-228.

12. Ksanfomality LV (2013) Possible signs of life on the planet venus. International Journal of Astronomy and Astrophysics 3: 57-79.

13. Ksanfomality LV (2013) Looking for mobility of hypothetical venusian fauna. Advances in Zoology and Botany 1: 25-29. 